\title{
Application Research on a Question-Answer Simulation Process Method in Complex Product Design
}

\author{
Ning Xiaobin ${ }^{1, *}$, Li Jie ${ }^{2}$, She Yini ${ }^{1}$, Fu Zhijun ${ }^{1}$ and Li Guang ${ }^{3}$ \\ ${ }^{1}$ College of Mechanical Engineering, Zhejiang University of Technology, Hangzhou, 310014, P.R. China; ${ }^{2}$ College of \\ Mechanical Engineering, Taiyuan University of Technology, Taiyuan, China, 030024; ${ }^{3}$ Technology Center, Taiyuan \\ Heavy Industry Co, Ltd., Taiyuan, 030024, P.R. China
}

\begin{abstract}
In order to support a question-answer driven simulation process, a design process model is introduced to describe problem statements, model specifications, simulation models and problem answers as separate objects. This method can effectively solve the engineering problems related to product design and technical performance, reduce modeling complexity and realize trace-ability on an object-to-object level of information. Then it conducts an applied study on performance matching of the hydraulic system in a large excavator. By the question-driven modular modeling, the working performance under lifting, digging and horizontal pushing operating condition, the power consumption of the hydraulic system and the influence of the performance matching parameters are simulated and analyzed. The results demonstrated that it was a practical valid method for process modeling of complex product, and it can accurately assess the excavating performance, provide the decision basis for the further design.
\end{abstract}

Keywords: Modeling and simulation, modular model architecture, question-answer driven, requirement specification, system model.

\section{INTRODUCTION}

In engineering design of complex products, it involves a lot of open and complex problems, which is a highly restricted and sophisticated cognitive reasoning of solving problem process. And the simulation modeling is a kind of activity includes analysis and comprehensive cognition reasoning. By the development of computer technology and the modeling and simulation software, it provides a new method for exploring and solving the design problems and extending the new technology of knowledge. At the same time, it is an iterative process for product requirement and problem solving in the whole process of product design. So it is crucial important for realizing the requirements specification and the trace-ability of the question-answer driven information [1].

Sellgren proposed and described the modular product modeling framework and information model based on the commercial software of CAD, CAE and PDM technology in detail. And the system configuration and application of model can assist the design reasoning and problem solving of the product. It can be targeted to solve specific problems by the model design reasoning method; however, it brought the complexity of the information management and tracing [2]. Malmqvist discussed a kind model for requirement driven product and process based on the chromosome product model which put forward by Andreasen [3], and proposed that the information granularity level of the management system is an important parameter which estimate the system has the trace-ability or not [4]. Almefelt et al. researched the requirement management system in the automobile industry application. It found that the requirements trace-ability is more complex and uncertain than the requirements management [5]. In order to ensure the trace-ability of information, it put forward three methods:

- Trace-ability tables

- Trace-ability lists

- Automated trace-ability links

Based on the literature $[6,7]$, this article introduces question-answer driven modeling and simulation method in the process of the design of complex product. And take the performance matching research for the large face-shovel hydraulic excavator attachment and hydraulic system as an example, through the system model configuration in a problem scene, it realizes and specifies the process of the model design reasoning, effectively solve the problem related to the product design reasoning, physical activity and technical performance, and ensure the trace-ability of the information object from the establishment of the requirements specification to the decision basis making process.

\section{THE DESIGN PROCESS MODEL}

The gateways management system [1], that is the new product development should be in charge of the gateways management according to the gateways structure chart of the product development chain, it makes the whole design process in effective control and supervision by continuously controlling each node in the process of the new product devel- 


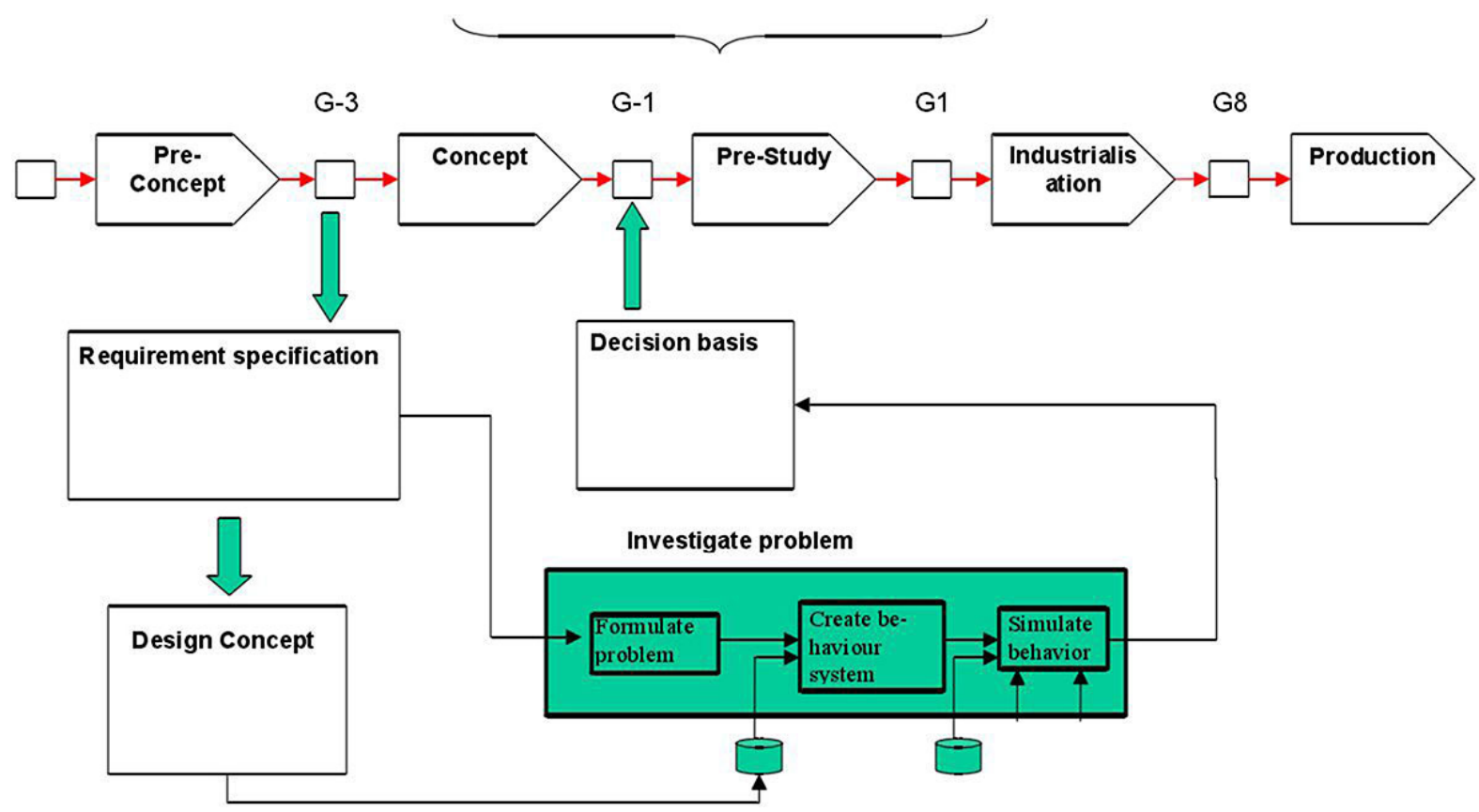

Fig. (1). Design process model of complex product.

opment. In order to improve the trace-ability and the verifiability of the requirement, this paper established a design process model as shown in Fig. (1)., which associated the model of the gateways management system with the general design process model put forward in literature [5]. It mainly consists of the stage of requirement specification, design concept, behavior model, and model verification. The information flow in Fig. (1) shows, the product concept can be further improved according to the requirements specification in a design phase, and then it gets the decision at gate G-3. This process will be completed in the product concept design phase. And it should be investigated in the activity "Investigate problem"; the decision basis from the activity is used for the next gate in the design process.

The activity "Investigate problem", which is the question-driven modeling, is the key to the verification of requirements specification. The approach to model-based design reasoning presented here is based on a modular model architecture, efficient system model configuration, and capture of the modeling rationale and process as engineering scenarios. A scenario is modeled as a recursive workflow (see Fig. 2) with six distinctive steps or scenes:

1. Define the context-dependent engineering problem and reformulate it as one or several question(s). The context may for example be a stored product model of an artifact;

2. Analyze each question and specify the requirements for a target model;

3. Synthesize (i.e., configure) a specific systems model that available knowledge suggests will satisfy the requirements;

4. Perform the simulation, or alternatively a set of simulations in probabilistic design, and store the result as an aggregated information object;
5. Analyze the simulation result to verify and validate $(V \& V)$ the model. Store them as information object;

6. Synthesize an answer from the analyzed simulation results (and identify new questions).

Each activity in a scenario needs input information, creates output information, is controlled by information, and is performed by an engineer with the aid of a mechanism or tool. Each of the scenario objects (i.e., the question, model requirements, model simulation result, and answer) has an owner attribute, a state attribute, and a causality relation to the object that was created in the immediately preceding activity in the scenario work-flow. This enables a granularity level of information that allows trace-ability on an object-toobject level between the attributes in the requirements specification and the estimated product properties.

\section{EXAMPLE}

The presented design process model can be used in a modeling and performance matching of the hydraulic system of the excavator working device, as shown in Fig. (3). It focuses on how to apply the iterative method in questionanswer driven process to build the co-simulation model of the hydraulic system of the working device and realize the performance optimization of the work device and the hydraulic system within the verification cycle process of requirements specification.

\subsection{Formulate Problem}

"Formulate problem", the first step in the verification of requirements specification, includes the following: the problem object description, application scenarios, requirement attributes, simulation model and problem answer. The information is stored as the problem object, as shown in Fig. (4). Among them, the requirement attributes is also been stored as the opposite object, as shown in Fig. (5). 


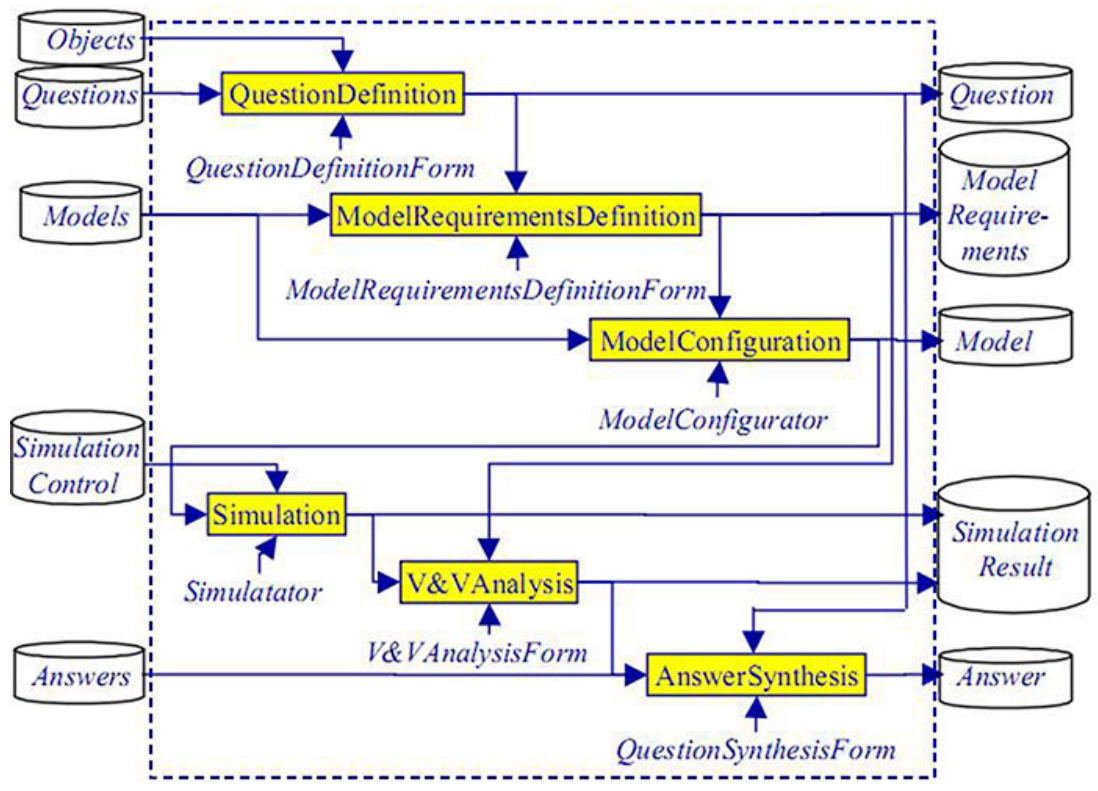

Fig. (2). Question-driven modeling activity of a scenario loop.

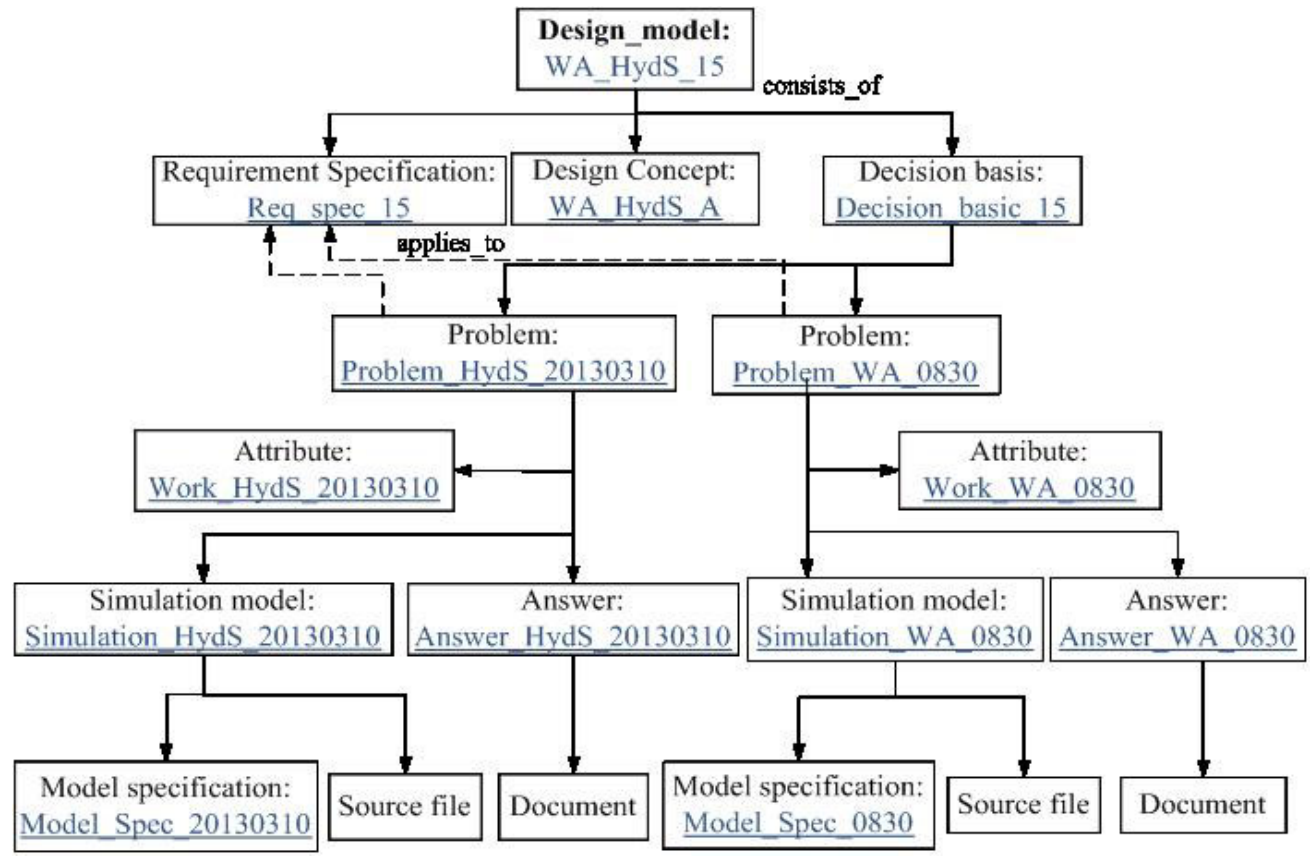

Fig. (3). A design model of the hydraulic system of the working device of the excavator.

\begin{tabular}{|c|l|}
\hline Name & Problem_HydS_20130310 \\
\hline Context & The hydraulic system of the work device \\
\hline Description & $\begin{array}{l}\text { How about the performance of lifting,digging and horizontal pushing conditions? } \\
\text { How about the performance parameters matching of the hydraulic system? }\end{array}$ \\
\hline Attribute & Work_HydS_20130310 \\
\hline Model Specification & Model_Spec_20130310 \\
\hline Simulation model & Simulation_HydS_20130310 \\
\hline Problem answer & Answer_HydS_20130310 \\
\hline
\end{tabular}

Fig. (4). Problem object. 


\begin{tabular}{|c|l|}
\hline Name & Work_HydS_20130310 \\
\hline Context & The hydraulic system of the work device \\
\hline Description & $\begin{array}{l}\text { The working performance of the hydraulic excavator in main conditions } \\
\text { The power consumption and efficiency of the hydraulic system }\end{array}$ \\
\hline Influence parameters & $\begin{array}{l}\text { the mining time, velocity of the work device, the pressure, power, flow range of } \\
\text { the hydraulic pump, the safety pressure of the main valve and hydraulic cylinder, etc. }\end{array}$ \\
\hline Image & Work_HydS.jpg \\
\hline Simulation model & Simulation_HydS_20130310 \\
\hline Problem answer & Answer_HydS_20130310 \\
\hline
\end{tabular}

Fig. (5). Requirement attribute object.

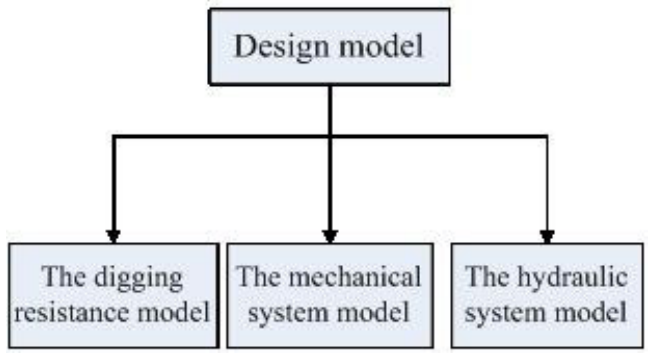

Fig. (6). The configuration structure of the system model.

\subsection{Create Behavior System}

Based on the problem object description, it is to define the model specification and establish the simulation model. The hydraulic excavator consists of mechanical simulation sub-model, hydraulic system sub-model of the working device, digging resistance model, as shown in Fig. (6).

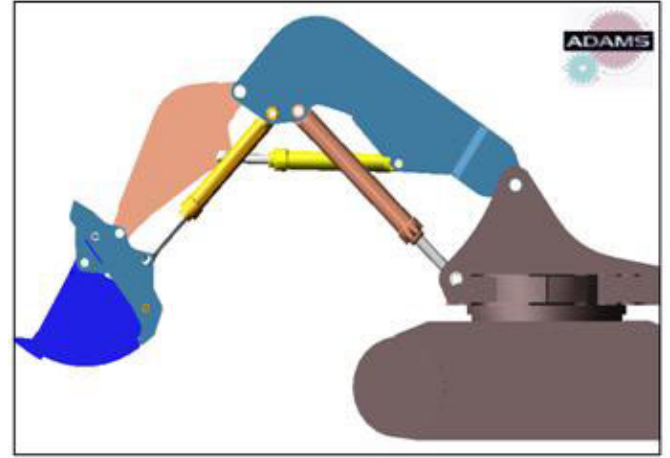

Fig. (7). The working device mechanical model in ADAMS.

The hydraulic excavator working device consists of the rotating platform, the bucket, the arm, the boom and the corresponding cylinders. To accurately simulate the working environment of the excavator, it is adopted the collaborative

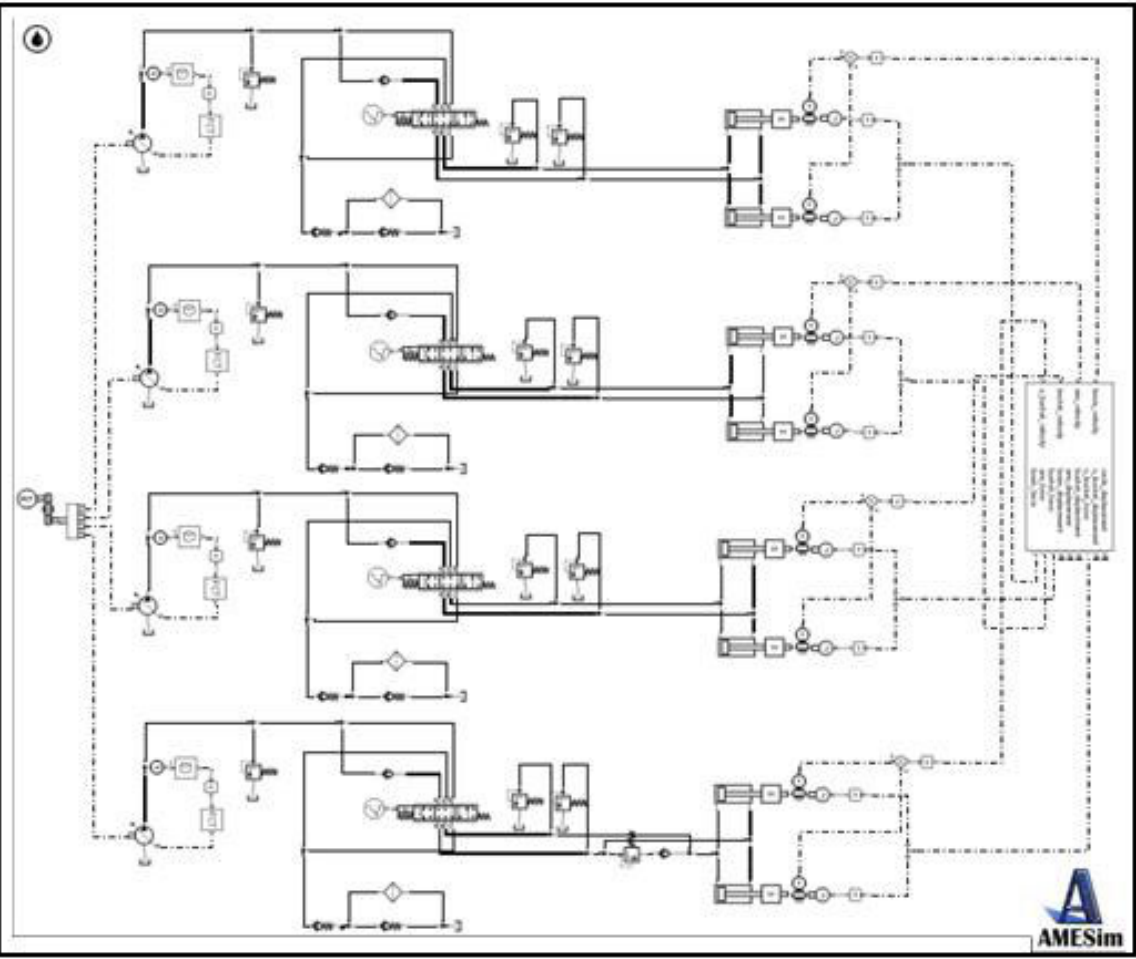

Fig. (8). The working device hydraulic system model in AMESim. 
simulation method. Firstly, the multi-rigid-body mechanical dynamics simulation model is built in the ADAMS, as shown in Fig. (7). Then, the hydraulic system of the working device of the excavator can use open loop system; mainly consist of hydraulic components of the variable hydraulic pump, multi-way valve, hydraulic cylinder, and the back pressure valve. It is established the hydraulic system model in the AMESim on the basis of the principle of the hydraulic system, as shown in Fig. (8). Finally, the co-simulation model is built by means of the interface module. Because of the complexity of the real load in the operating process of the excavator, here it is established the ore particle model in the EDEM by using the discrete element method, as shown in Fig. (9). The accurate and reliable of the digging resistance curve is got, and can be uploaded in the dynamic simulation model. The defined model specification object and the simulation model object in the activity "Create behavior system" is shown in Figs. (10) and (11).

\subsection{Simulate Behavior}

The hydraulic system model is built in the AMESim, through the interface module the co-simulation model is got. Under a variety of load conditions, the excavating load curve, which is obtained in the EDEM, is uploaded in the Adams. The simulation parameters of hydraulic components are set, and then it set up the simulation time, the communication interval, the integral form and the operation type etc. in the running model. Finally, the simulation result is obtained.

According to the requirement attribute of the problem object "Problem HydS 20130310", in this article it carries out simulation analysis for the mining performance of the work device and the dynamic matching for the hydraulic system. It can be got answers from the simulation results, and provide decision basis for optimization design further.

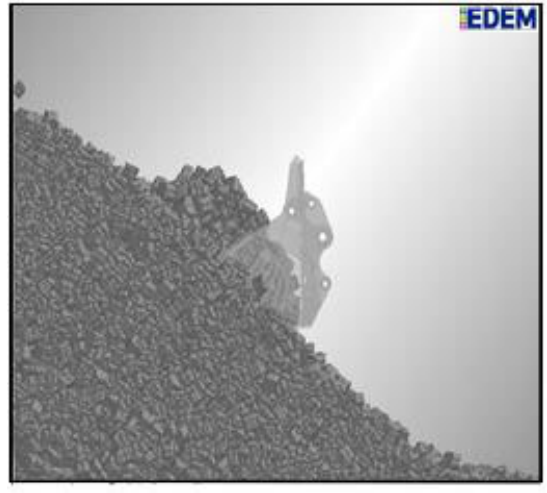

Fig. (9). The digging resistance model in EDEM.

\subsubsection{Performance Simulation Analysis of the Work De- vice in the Boom Lifting Condition}

The boom lifting is which control the hydraulic cylinder of the boom through the confluence of the multi-way valve. At the same time, the hydraulic cylinder of the arm and bucket is closed. The whole process of simulation is as follows: at first, the hydraulic boom and arm cylinders keep the shrinkage state; the bucket cylinder extends $1 \mathrm{~m}$. And then, the boom cylinder extends from the shrinkage to the fully open in no-load and full-load conditions. In there, no-load means no ore material inside the bucket and full-load refers loaded with 30 tons of ore material in the bucket. The trajectory of the bucket tip in the boom lifting process is shown in Fig. (12). The simulation results as shown in Figs. (13-16).

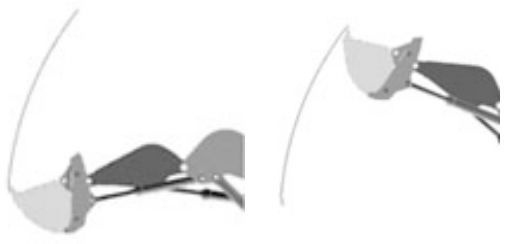

Fig. (12). The trajectory of the bucket tip in the boom lifting process.

\begin{tabular}{|c|l|}
\hline Name & Model_Spec_20130310 \\
\hline Context & The hydraulic system of the work device \\
\hline Description & $\begin{array}{l}\text { Analyze the working performance of the work device and the load } \\
\text { effect on the performance of the hydraulic system }\end{array}$ \\
\hline Problem & Problem_HydS_20130310 \\
\hline Target application & AMESim ADAMS EDEM \\
\hline Submodel & $\begin{array}{l}\text { the hydraulic system model, the kinematic model of the work device, } \\
\text { the digging resistance model }\end{array}$ \\
\hline
\end{tabular}

Fig. (10). Model specification object.

\begin{tabular}{|c|l|}
\hline Name & Simulation_HydS_20130310 \\
\hline Context & The hydraulic system of the work device \\
\hline Description & $\begin{array}{l}\text { The analysis of the working performance of the work device and the load } \\
\text { effect on the performance of the hydraulic system }\end{array}$ \\
\hline Model specification & Model_Spec_20130310 \\
\hline Simulation file & Analy_HydS_20130310 \\
\hline
\end{tabular}

Fig. (11). Simulation model object. 


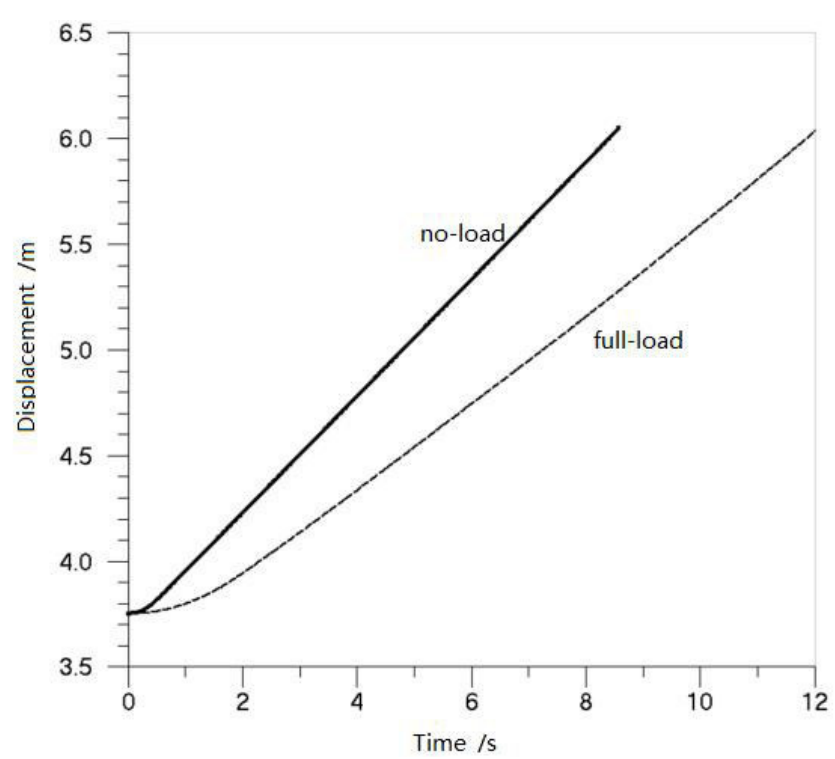

Fig. (13). The boom cylinder displacement curve in the lifting process.

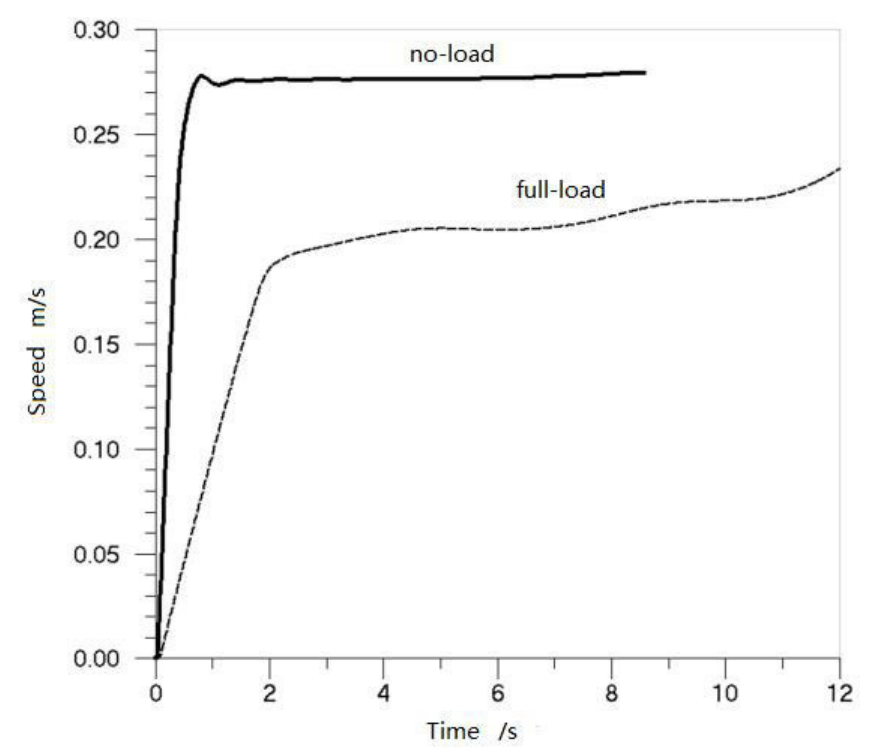

Fig. (14). The boom cylinder speed curve in the lifting process.

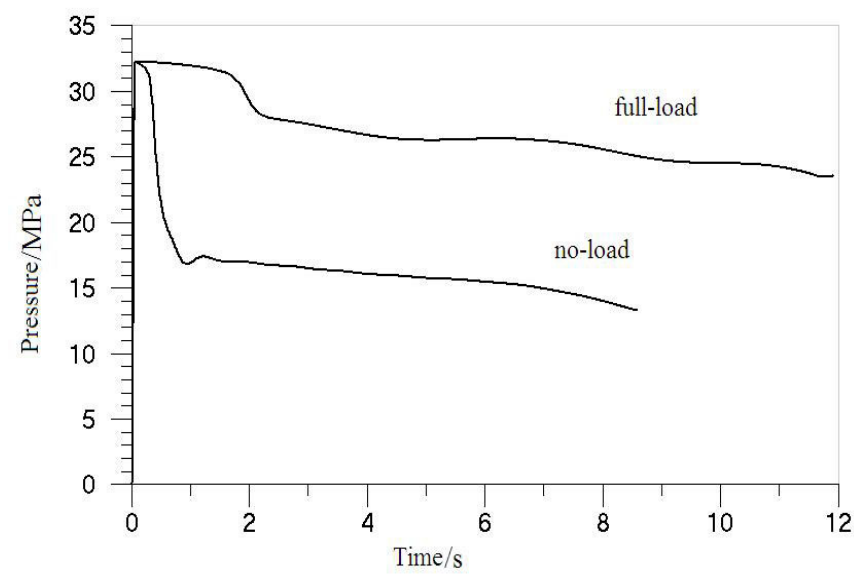

Fig. (15). The boom cylinder pressure curve in lifting process.

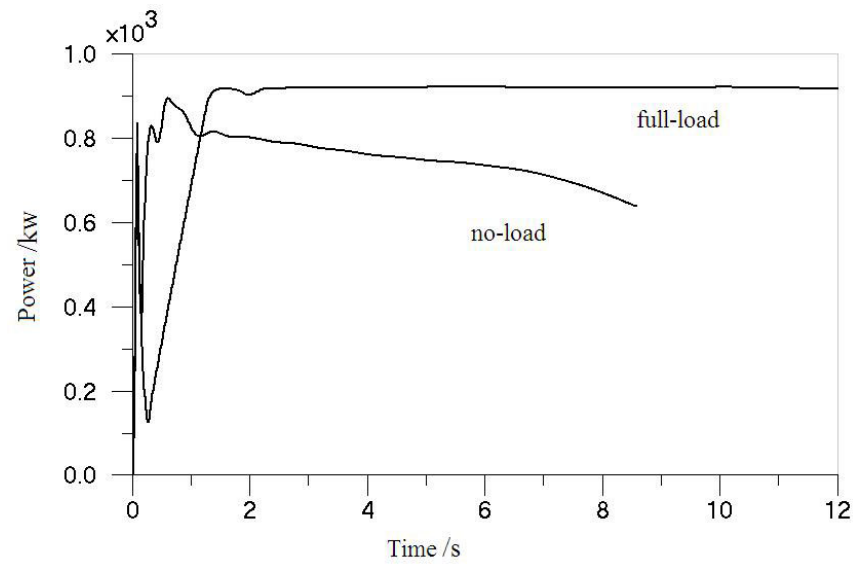

Fig. (16). The power consumption curve of the hydraulic pump in lifting process.

According to the Figs. (13 and 14), in no-load condition, the hydraulic cylinder of the boom should be $8.5 \mathrm{~s}$ to the lifting highest point. The speed reaches to the largest $0.28 \mathrm{~m} / \mathrm{s}$ in lifting process; in full-load condition, because of the quick and unexpected change in velocity at the start-up process, the hydraulic system will produce pressure impact. In order to reduce pressure impact, it makes the velocity of hydraulic cylinder rising slowly and takes $12 \mathrm{~s}$ to the highest point. The speed reaches to the largest $0.22 \mathrm{~m} / \mathrm{s}$ in lifting process. From Fig. (15) shows, no matter the hydraulic excavator is in noload or full-load condition, the pressure of the boom cylinder reaches a maximum value $32 \mathrm{MP}$, at the initial stage. And with the increase in lifting height, the pressure of the boom cylinder decreases gradually. In no-load condition, the boom is lifted to the highest in $8.5 \mathrm{~s}$, the pressure inside the boom cylinder is $13 \mathrm{MPa}$; in the full-load condition, the boom is lifted to the highest in $12 \mathrm{~s}$, the pressure is $27 \mathrm{MPa}$. Therefore, during the boom lifting process, the change of the pressure in the boom cylinder did not exceed the maximum working pressure; the hydraulic system can meet the demand of the lifting boom. In the initial stage of boom lifting mechanism, because of greater moment of inertia, stress is also larger; but with the rising of the boom, the torque taken by the gravity of the mechanism on the hinge joint of the boom is reduced, the force of the hydraulic cylinder also gradually reduced. The pressure change for each hydraulic cylinder and the power consumption can meet the design requirements in the lifting process, as shown in Fig. (16).

\subsubsection{Performance Simulation Analysis of the Work De- vice in the Digging Condition}

The hydraulic excavator works mainly by the way of arm mining or compound mining. In the stage of arm mining, the hydraulic cylinder of the arm is supplied by four main pumps; the hydraulic cylinder of the boom is closed. At the same time, it should keep the direction of the digging force along the external surface of the bucket lip by adjusting the posture of the bucket. The process of simulation is that the hydraulic excavator work by arm mining, the hydraulic cylinder of the arm extends to the fully open and the bucket keeps the optimal angle.

For arm mining, it is analyzed the power, the pressure, the flow of the hydraulic system and the parameter matching 


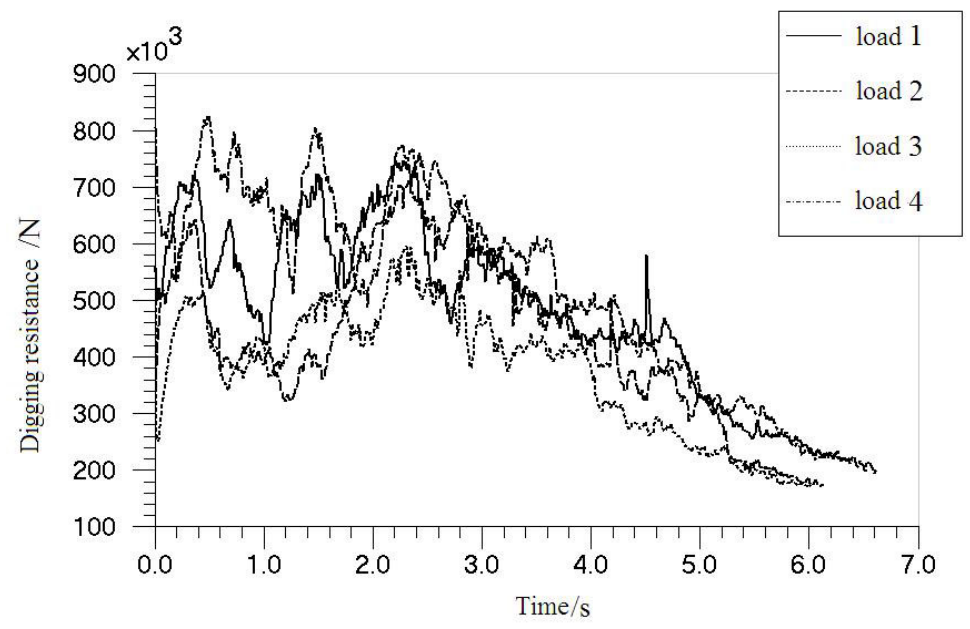

Fig. (17). EDEM digging resistance in different dipping depth.

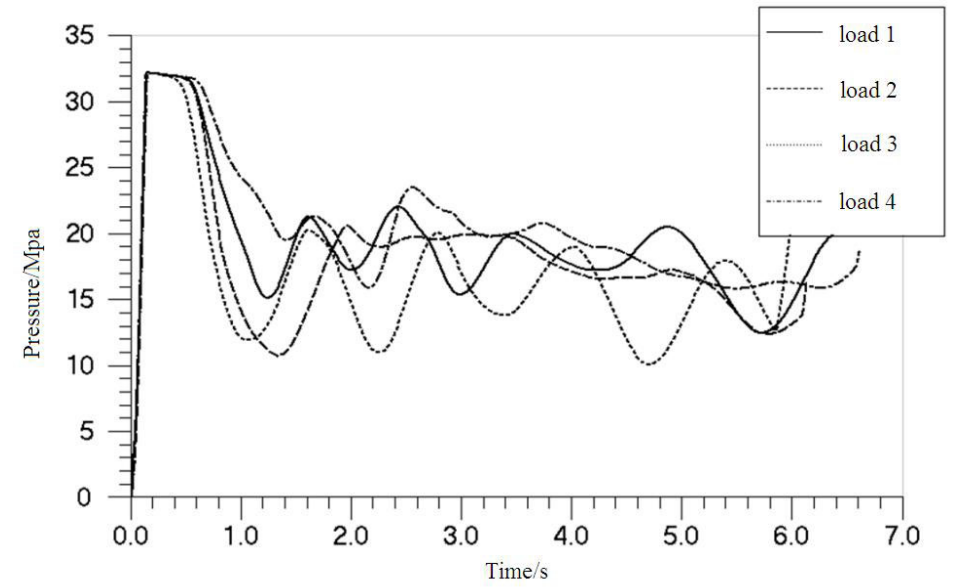

Fig. (18). The pressure change of the arm cylinder in different load conditions.

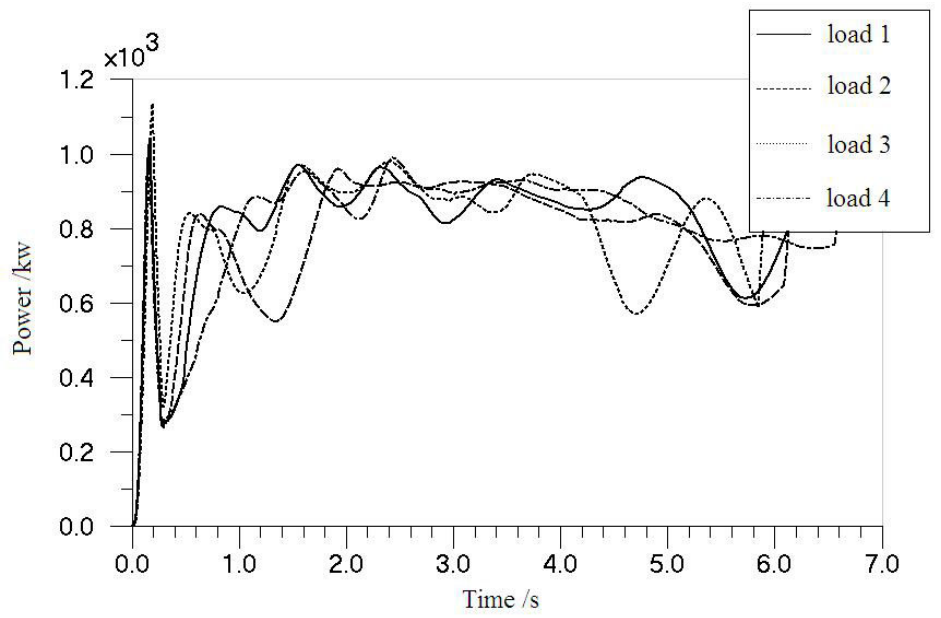

Fig. (19). The power change of the hydraulic pump in different load conditions.

for each hydraulic component. As shown in Fig. (17), the digging resistance of the excavator makes larger with the increase of the digging depth on ore piles in the process of the arm mining. From the Figs. (18) and (19), it is analyzed that the pressure of the hydraulic cylinder of the arm has some fluctuation, but is less than a limited pressure $32 \mathrm{MPa}$. And the other closure pressure of the hydraulic cylinder is controlled within a given range. The hydraulic pump can adjust the flow with constant power control, it makes full use of the power of the hydraulic pump and the mining velocity can meet the actual requirement. The hydraulic pump power, the pressure, flow rate changes of each hydraulic cylinder can satisfy the different load conditions and meet the design requirement. 


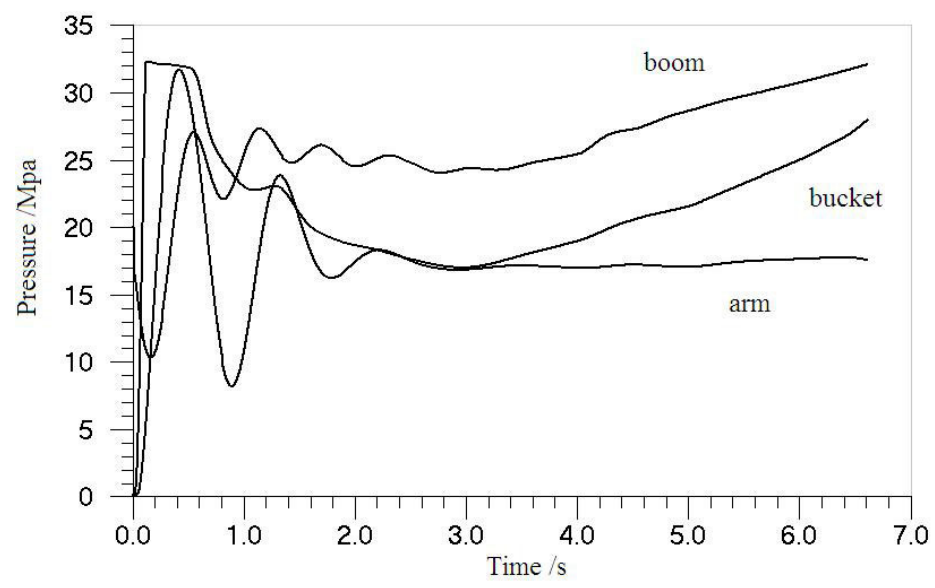

Fig. (20). The pressure curve of each hydraulic cylinder in the horizontal pushing condition.

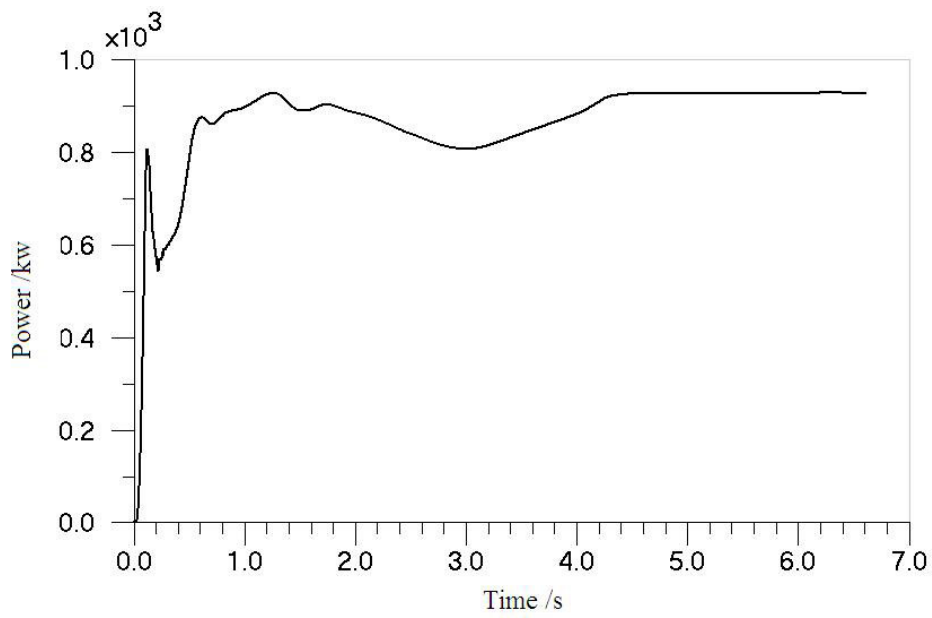

Fig. (21). The power consumption curve in the horizontal pushing condition.

\subsubsection{Performance Simulation Analysis of the Work De- vice in Horizontal Push-and-Press Condition}

The hydraulic excavator makes use of the cooperation with the boom cylinder, the arm cylinder and the bucket cylinder to carry on the horizontal push-and-press action. In the process, the boom cylinder is supplied by a pump, the arm cylinder is supplied by two pumps, and the bucket cylinder is supplied by a pump. It works by operating the handle and the multi-way valve. The working time of the arm cylinder extending to fully open, the speed change of each hydraulic cylinder, etc., are obtained by simulation. The simulation shows that it realizes horizontal push-and-press digging by the joint action of each hydraulic cylinder to control the flow of the pump. And the pressure change of the hydraulic cylinder and the output power and the hydraulic pump meet the design requirement. So, from the efficiency and power consumption analysis, it realize the performance matching requirements between the working device and the hydraulic system of the hydraulic excavator, as shown in Figs. (20) and (21).

\subsubsection{Performance Simulation Analysis of the Hydraulic Excavator Typical Working Cycle}

Hydraulic excavator typical working cycle can be divided into four stages. The first stage is the process that after reaching the initial position and adjusting the initial excavation bucket posture, the hydraulic excavator began to digging. The second stage refers to the entire stick digging process. The third stage is the process that the full bucket is lifted and swung to the unloading position. And the final stage means the process that ores are discharged from the bucket, and then an empty bucket return to the next work cycle. The co-simulation method is used in the process of the typical work cycle to calculate and analyze performance parameters of the hydraulic system and the work device load condition.

According to the Figs. (22) and (23), In the first stage, hydraulic excavators can reach to the initial digging position by adjusting the length of hydraulic cylinders piston rod. The second stage starts at $6.5 \mathrm{~s}$ and ends at $13 \mathrm{~s}$. The maximum flow rate reaches $0.28 \mathrm{~m} / \mathrm{s}$. After ending the process of the stick digging, the full bucket is lifted and swung to the specified uploading height location in $7.5 \mathrm{~s}$. And the lifting speed can reach to the $0.22 \mathrm{~m} / \mathrm{s}$. In the entire lifting process, the bucket digging angle must be maintained an optimal angle to avoid the material spilled. At last, the finial stage starts, each hydraulic cylinder is retracted into the next work cycle.

As can be seen from Fig. (22), in the boom cylinder retraction process, the speed is too large, which will cause 


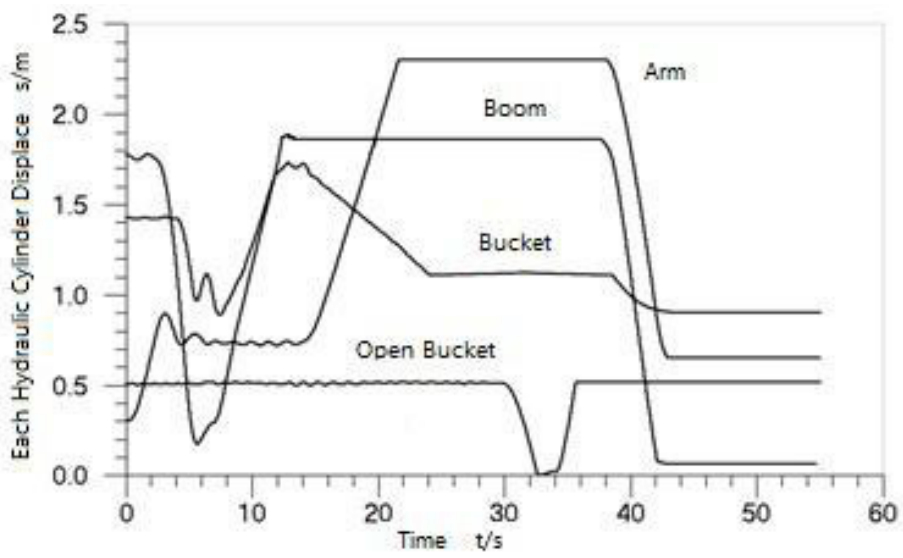

Fig. (22). Each hydraulic cylinder displacement curve.
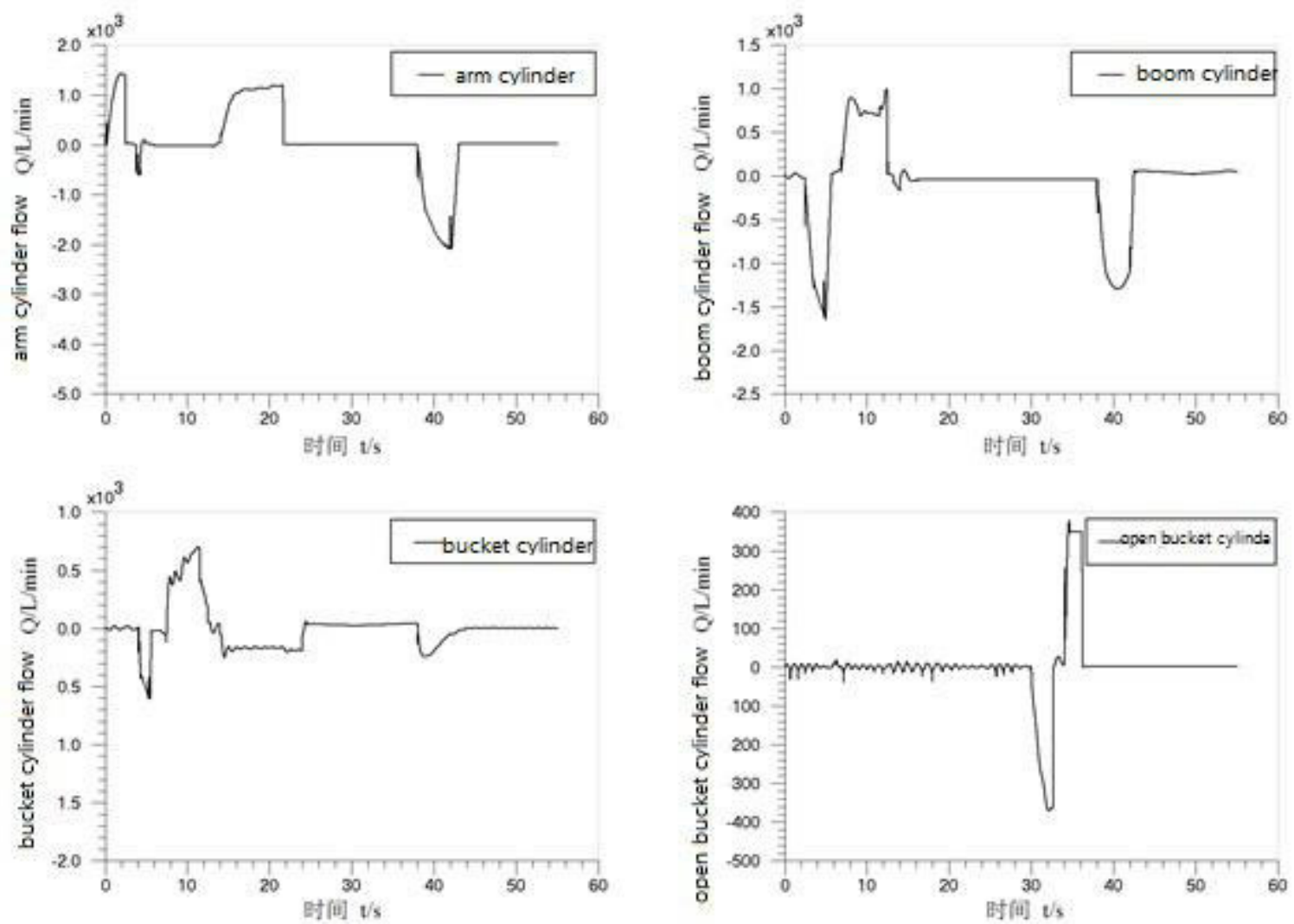

Fig. (23). Each hydraulic cylinder rod chamber flow curve.

cavitation phenomenon in rod chamber. At the same time, in order to avoid increasing the back pressure caused by the waste of energy, the flow of the boom cylinder regeneration circuit is designed in the fall process. During the working course, the multi-way valve commutation position need to be constantly switched. Due to the opening and closing action fast simulation process, when a sudden change in direction, it will cause some pressure shocks, as shown in Fig. (23), so the commutation action to slow the action, to avoid pressure shock.

The entire work cycle simulation results showed that the pressure change parameters of each hydraulic pump, multivalve and hydraulic cylinder can meet the excavators working requirements, and system performance parameters to

match the overall design is reasonable. And it is reasonable that the match of the hydraulic performance to the whole excavator design performance. At the same time, the retraction speed, mining time, demand flow distribution and other operating parameters of each hydraulic cylinder also achieve the actual digging needs Fig. (24).

The above analysis shows that, the optimal hinge points design program of the working device meets the performance requirement of the working device under the typical working conditions such as arm digging, boom lifting, horizontal pushing and the typical working cycle. The answer to the raised question in the simulation results and the object description of formulate problem should be stored in a diagram form, as shown in Fig. (25). 

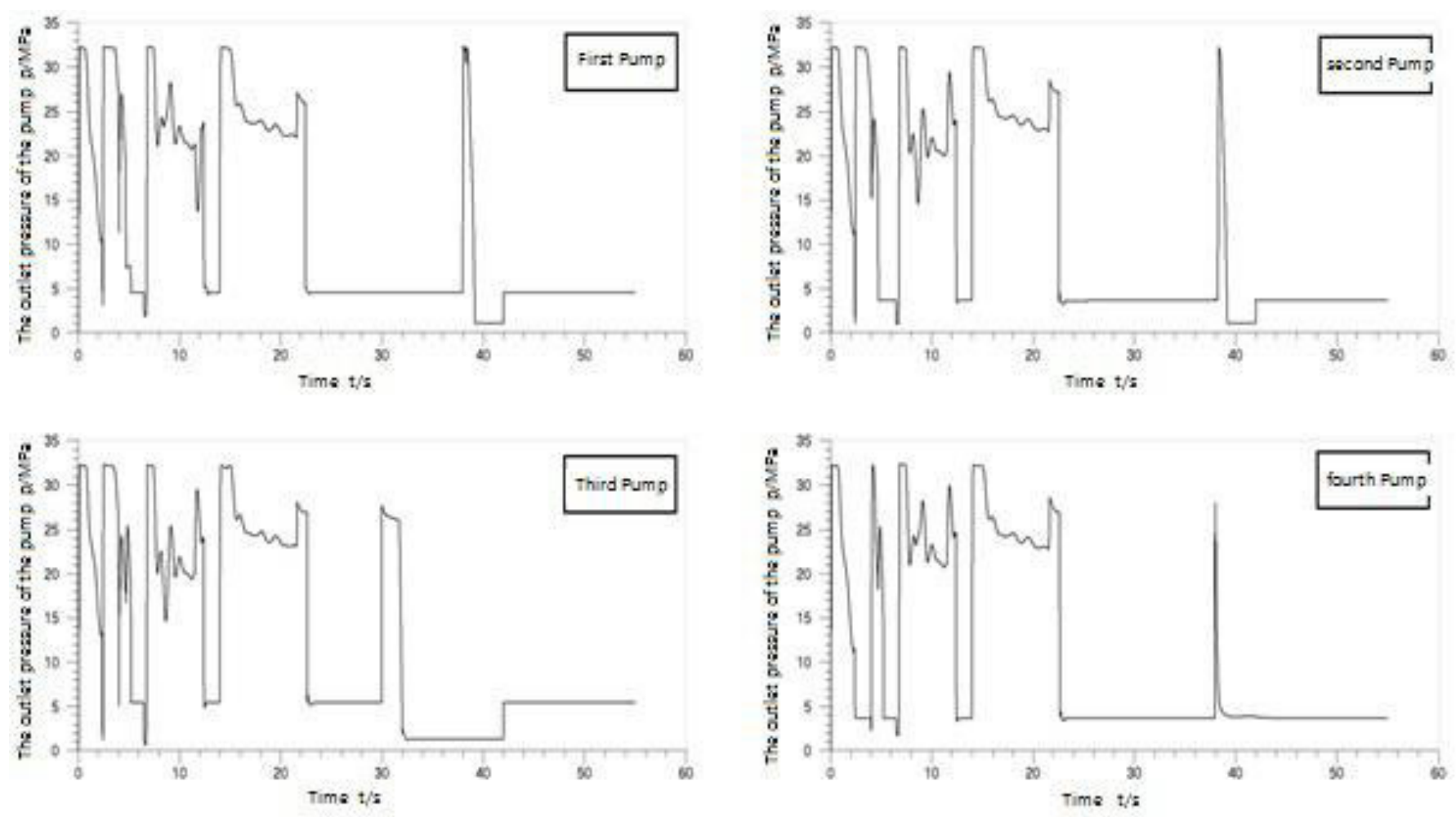

Fig. (24). Each pump outlet pressure curve.

\begin{tabular}{|c|l|}
\hline Name & Decision_basis_20130310 \\
\hline Description & $\begin{array}{l}\text { Decision basis for performance optimization of the hydraulic } \\
\text { system of the work device }\end{array}$ \\
\hline General attributes & the boom lifting, the arm digging, the horizontal pushing, etc. \\
\hline Minimum digging time & $6 \mathrm{~s}$ \\
\hline Maximum digging velocity & $0.4 \mathrm{~m} / \mathrm{s}$ \\
\hline The pressure changes & Graph1 \\
\hline The power consumption & Graph2 \\
\hline Requirement attribute & Work_HydS_20130310 \\
\hline Decision basis & Problem_HydS_20130310 \\
\hline
\end{tabular}

Fig. (25). Decision basis object.

\section{CONCLUSION}

1. This proposed design method is mainly used for the conceptual design of complex products. By importing formulate problem, behavior model, simulation model and decision basis in the design process model, it realizes the object-object information granularity level between product attributes and requirement specification attributes through taking question-answer driven iteration verification solution for product requirement specification. The example shows that the method is practicable and the performance of the designed excavator work device meets the design requirement.

2. It implements the transformation from the requirement to the simulation model, and provides the possibility for the study of the requirement change effects on the per- formance of the product. When requirements change, part of the simulation model and the results can be reused, reduce the repetition of the modeling work.

3. This method is applied to the performance matching for the working device and the hydraulic system of the large face-shovel hydraulic excavator. It has realized the trace-ability of the information object in the simulation modeling and the accurate assessment of the performance of the hydraulic system. It provides the decision basis for the product design further.

\section{CONFLICT OF INTEREST}

The authors confirm that this article content has no conflict of interest. 


\section{ACKNOWLEDGEMENTS}

Project supported by the National Natural Science Foundation of China (Grant No. 51375452).

\section{REFERENCES}

[1] H. Liu, G. Qi, T. Zhang, and Y. Ji, "Research on multi-disciplinary process modeling of conceptual design for complex product", Journal of Zhejiang University (Engineering Science), vol. 43, no. 3, pp. 517-521, 2009.

[2] U. Sellgren, "Architecting models of technical systems for nonroutine simulations", In: Proceedings International Conference on Engineering Design, Stockholm, Sweden, 2003, pp. 32-35.
[3] M. M. Andreasen, "Designing on a Designer's Workbench (DWB)", In: Proceedings of the $9^{\text {th }}$ WDK Workshop, Rigi, Switzerland, 1992.

[4] J. Malmqvist, "Implementing requirements management - a task for specialized software tools or PDM systems", Systems Engineering, vol. 4, no. 1, pp. 49-57, 2001.

[5] L. Almefelt, F. Andersson, P. Nilsson, and J. Malmqvist, "Exploring requirements management in the automotive industry", In: Proceedings International Conference on Engineering Design, Stockholm, Sweden, 2003, pp. 19-21.

[6] Y. She, X. Ning, and Q. Lin, "Simulation in complex construction vehicle design based on an iterative question-answer drive process", China Mechanical Engineering, vol. 25, no. 8, pp. 1096-1100, 2014.

[7] X. Ning, Y. She, and A. Li, "Design in face-shovel hydraulic excavator based on question-answer drive", Journal of Applied Sciences, vol. 13 , no. 12 , pp. 2196-2202, 2013.

Received: June 10, 2015

(C) Xiaobin et al.; Licensee Bentham Open.

This is an open access article licensed under the terms of the (https://creativecommons.org/licenses/by/4.0/legalcode), which permits unrestricted, noncommercial use, distribution and reproduction in any medium, provided the work is properly cited. 